\title{
SPECTRAL CONCENTRATION AND VIRTUAL POLES. II
}

\author{
BY
}

JAMES S. HOWLAND( $\left.{ }^{1}\right)$

\begin{abstract}
Spectral concentration at an isolated eigenvalue of finite multiplicity of the selfadjoint operator $H_{\varepsilon}=T_{\varepsilon}+A_{\varepsilon} B_{\varepsilon}$ is shown to arise from a pole of an analytic continuation of $A_{\varepsilon}\left(H_{\varepsilon}-z\right)^{-1} B_{\varepsilon}$. An application to quantum mechanical barrier penetration is given.
\end{abstract}

This article is concerned with the problem of isolated eigenvalues of finite multiplicity which disappear into a continuous spectrum on perturbation. We wish to determine conditions under which spectral concentration results from a virtual pole of the resolvent $R_{\varepsilon}(\zeta)$ of the perturbed operator $H_{\varepsilon}$; that is, from a pole of an analytic continuation of certain matrix elements of $R_{\varepsilon}(\zeta)$. In [5], we have reviewed previous work, and have discussed the case in which the unperturbed operator $H_{0}$ is of finite rank.

The present article is based on the factorization technique [6], [7], [8]. We proceed as follows. We do not divide the perturbed operator in the usual manner as

$$
H_{\varepsilon}=H_{0}+V_{\varepsilon}, \quad \varepsilon>0,
$$

where $V_{\varepsilon}$ is the perturbation, but rather as

$$
H_{\varepsilon}=T_{\varepsilon}+A_{\varepsilon} B_{\varepsilon}, \quad \varepsilon>0,
$$

where $H_{\varepsilon}$ and $T_{\varepsilon}$ are close in the sense that $Q_{\varepsilon}(z)=A_{\varepsilon}\left(T_{\varepsilon}-z\right)^{-1} B_{\varepsilon}$ is compact, and has an analytic continuation $Q_{\varepsilon}^{+}(z)$ from the upper half-plane across the axis at the eigenvalue $\lambda_{0}$ in question. The idea, which is implicit in [5], is that $H_{\varepsilon}$ and $T_{\varepsilon}$ are quite similar, where as $H_{\varepsilon}$ and $H_{0}$ are not. In fact, in [5], we chose $A_{\varepsilon} B_{\varepsilon}=H_{0}$. Of course, the perturbation-theoretic philosophy here is that $T_{\varepsilon}$ is a family of unperturbed operators, for which $\left(T_{\varepsilon}-z\right)^{-1}$ and other such quantities are computable, so that conditions may reasonably be placed on $T_{\varepsilon}$ which cannot be placed on $H_{\varepsilon}$. Our main result $(\S 2)$ states that, under suitable conditions, there is spectral concentration associated with poles of $\left[I+Q_{\varepsilon}^{+}(z)\right]^{-1}$ in the usual manner. We apply this $(\S 3)$ to improve the results of [5] by eliminating a nondegeneracy assumption and $(\$ 4)$ to consider a problem of quantum mechanical barrier penetration, such as is usually discussed by means of the WKB method.

Received by the editors May 25, 1970.

AMS 1969 subject classifications. Primary 4748; Secondary 4760, 8134, 8147.

Key words and phrases. Spectral concentration, virtual pole, perturbation theory, Schroedinger equation.

(') Supported by ARO Grant DA-ARO-D-31-124-G978.

Copyright (C) 1972, American Mathematical Society 
This paper is independent of the previous article [5] of the same title.

Let $\sigma(T), \rho(T)$ and $\mathscr{D}(T)$ denote the spectrum, resolvent set and domain of the operator $\boldsymbol{T}$. $\boldsymbol{C}$ and $\boldsymbol{R}$ denote the complex and real numbers.

1. Hypotheses and preliminaries. We shall first recall some results of [6].

Hypothesis I. Let $\mathscr{H}$ and $\mathscr{H}^{\prime}$ be separable Hilbert spaces. Let $\mathrm{T}$ be a selfadjoint operator on $\mathscr{H}$ with resolvent $G(z)=(T-z)^{-1}$, and let $A$ and $B$ be closed, densely defined operators from $\mathscr{H}$ to $\mathscr{H}^{\prime}$. Assume that

(a) $\mathscr{D}(T) \subset \mathscr{D}(A) \cap \mathscr{D}(B)$ and $(A x, B y)=(B x, A y)$ for every $x, y \in \mathscr{D}(A) \cap \mathscr{D}(B)$.

(b) For every $z \in \rho(T)$, the operator $A G(z) B^{*}$, which is defined on $\mathscr{D}\left(B^{*}\right)$, has a compact extension $Q(z)$ to $\mathscr{H}^{\prime}$.

1.1. Lemma [6], [7], [9]. If Hypothesis I is satisfied, then

(a) There exists a selfadjoint extension $H$ of $T+B^{*} A$ such that if $z \in \rho(T)$ and $I+Q(z)$ has a bounded inverse, then $z \in \rho(H)$ and

(1.1) $R(z)=G(z)-[B G(\bar{z})]^{*}[I+Q(z)]^{-1} A G(z)$ where $R(z)=(H-z)^{-1}$.

(b) $\mathscr{D}(H) \subset \mathscr{D}(A)$ and $A R(z) B^{*}$ has a compact extension $Q_{1}(z)$ from $\mathscr{D}\left(B^{*}\right)$ to $\mathscr{H}^{\prime}$, which is such that

(1.2) $I-Q_{1}(z)=[I+Q(z)]^{-1}$ for every $z \in \rho(T)$ where the right side of $(1.2)$ exists.

(c) $T$ arises from $H$ in the same manner; that is,

(1.3) $G(z)=R(z)+[B R(\bar{z})]^{*}\left[I-Q_{1}(z)\right]^{-1} A R(z)$.

(The signs change, since $T$ extends $H-B^{*} A$.)

HYPOTHESIS II. Let $\Omega$ be a domain in $C$ which intersects the real axis, and define $\Omega^{ \pm}=\{z \in \Omega: \pm \operatorname{Im} z>0\}$. Then $Q(z)$ is analytic on $\Omega^{ \pm}$. Assume that $Q(z)$ has an analytic continuation $Q^{ \pm}(z)$ from $\Omega^{ \pm}$to all of $\Omega$.

Note that $Q^{+}(z)$ and $Q^{-}(z)$ need not agree on $\Omega$.

It follows ([6, Lemma 1.5], [10] and [11, Theorem 1]) that $Q_{1}(z)$ has a meromorphic continuation from $\Omega^{ \pm}$to $\Omega$ given by

$$
I-Q_{1}^{ \pm}(z)=\left[I+Q^{ \pm}(z)\right]^{-1}
$$

and that the principal part of $Q_{1}^{ \pm}(z)$ at each of its poles has finite rank.

1.2. TheOREM. If Hypotheses I and II hold, then there exists a subspace $\mathscr{M}$ of $\mathscr{H}$ reducing both $T$ and $H$, such that

(a) $T=H$ on $\mathscr{M}^{\perp}$,

(b) the parts of $T$ and $H$ in $\mathscr{M}$ are absolutely continuous, except that $H$ has eigenvalues of finite multiplicity at the real poles of $Q_{1}^{ \pm}(z)$.

This result follows directly from Theorem 3.1 of [6], which also gives a formula for the multiplicity of the eigenvalues of $H$.

The following is the main hypothesis of this paper.

HyPOTHESIS III. For $n \geqq 0$, let $T_{n}, A_{n}$ and $B_{n}$ satisfy Hypotheses I and II on a domain $\Omega$ independent of $n$. Assume that

(a) $Q^{ \pm}(z, n) \rightarrow Q^{ \pm}(z, 0)$ uniformly on $\Omega$, in operator norm.

(b) $Q_{1}^{ \pm}(z, 0)$ is analytic on $\Omega$, except for a nontrivial pole at a real point $\lambda_{0}$ of $\Omega$. 
(c) There exists a dense subspace $\mathscr{D}$ of $\mathscr{H}^{\prime}$ such that $\mathscr{D} \subset \mathscr{D}\left(A_{n}^{*}\right) \cap \mathscr{D}\left(B_{n}^{*}\right)$ for every $n \geqq 0$, and $A_{n}^{*} x \rightarrow A_{0}^{*} x$ and $B_{n}^{*} x \rightarrow B_{0}^{*} x$ strongly, for every $x \in \mathscr{D}$.

(d) For $\operatorname{Im} z \neq 0, G_{n}(z), A_{n} G_{n}(z)$ and $B_{n} G_{n}(z)$ converge strongly to $G_{0}(z), A_{0} G_{0}(z)$ and $B_{0} G_{0}(z)$ respectively.

(e) $\lambda_{0}$ is not an eigenvalue of $T_{0}$.

Notation. We shall write $G_{n}(z)=\left(T_{n}-z\right)^{-1}, R_{n}(z)=\left(H_{n}-z\right)^{-1}, T_{n}=\int \lambda d E_{n}(\lambda)$, $H_{n}=\int \lambda d E_{n}^{(1)}(\lambda)$, where $H_{n}$ is the operator obtained from $T_{n}, A_{n}$ and $B_{n}$ according to Lemma 1.1. $Q(z, n)$ is the extension of $A_{n} G_{n}(z) B_{n}^{*}$, and $Q^{ \pm}(z, n)$ its analytic continuation. Similarly for $Q_{1}^{ \pm}(z, n)$.

By Theorem 1.2, $\lambda_{0}$ is an eigenvalue of $H_{0}$ of finite multiplicity $m$. If $P_{0}$ is the projection onto $\operatorname{ker}\left(H_{0}-\lambda_{0}\right)$, then [6, Theorem 3.1]

(1.4) $Q_{1}^{ \pm}(z, 0)=\left(z-\lambda_{0}\right)^{-1} A P_{0}\left[B P_{0}\right]^{*}+L_{0}^{ \pm}(z)$ where $L_{0}^{ \pm}(z)$ is analytic on $\Omega$.

Hypothesis III(d) is used only in the following proof.

1.3. LEMMA. If $\operatorname{Im} z \neq 0$, then $R_{n}(z) \rightarrow R_{0}(z)$ strongly.

Proof. If $x \in \mathscr{D}$, then $\left[B_{n} G_{n}(\bar{z})\right]^{*} x=G_{n}(z) B_{n}^{*} x$ converges to $G_{0}(z) B_{0}^{*} x=\left[B_{0} G_{0}(\bar{z})\right]^{*} x$. Since the norms of $B_{n} G_{n}(z)$ are bounded, we have $\left[B_{n} G_{n}(\bar{z})\right]^{*} \rightarrow\left[B_{0} G_{0}(\bar{z})\right]^{*}$ strongly. The result now follows from formulas (1.1) and (1.2).

The next lemma holds for operator-valued functions on an arbitrary Banach space, and is due essentially to Steinberg [11].

1.4. Lemma. Let $T_{n}(z)$ be a sequence of analytic functions on a domain $\Omega$, with values in the compact operators, such that $T_{n}(z) \rightarrow T_{0}(z)$ uniformly on $\Omega$. Let $z_{0} \in \Omega$. Then there exists a neighborhood $D$ of $z_{0}$, and analytic functions $A_{n}(z), U_{n}(z)$ and $F_{n}(z), n \geqq 0$, such that

(a) $A_{n}(z)$ and $U_{n}(z)$ are invertible for each $z \in D$, and $U_{0}\left(z_{0}\right)=I$.

(b) $U(z) F(z) U^{-1}(z)$ is reduced by $\operatorname{ker}\left(I+T_{0}(0)\right)$ and vanishes on a complementary subspace of $\operatorname{ker}(I+T(0))$.

(c) $A_{n}(z)\left(I+T_{n}(z)\right)=I+F_{n}(z), n \geqq 0$, and

(d) $A_{n}(z), U_{n}(z)$ and $F_{n}(z)$ converge to $A_{0}(z), U_{0}(z)$ and $F_{0}(z)$ uniformly on $D$.

The following theorem describes the singularities of $Q_{1}^{ \pm}(z, n)$ near $\lambda_{0}$.

1.5. TheOREM. If Hypothesis III is satisfied, then there exists a neighborhood D of $\lambda_{0}$ such that for $n$ sufficiently large there are (counting multiplicities) no more than $m$ poles of $Q_{1}^{ \pm}(z, n)$ in $D$, all of which converge to $\lambda_{0}$ as $n$ tends to infinity.

Proof. By Lemma 1.4, $A_{n}(z), U_{n}(z)$ and $D$ may be chosen such that

$$
A_{n}(z)\left(I+Q^{ \pm}(z, n)\right)=I+F_{n}^{ \pm}(z)
$$

where $U_{n}(z) F_{n}^{ \pm}(z) U_{n}^{-1}(z)$ is an operator on $\operatorname{ker}\left(I+Q^{ \pm}\left(\lambda_{0}, 0\right)\right)$. It follows (cf. [11, Theorem 1]) that the poles of $Q_{1}^{+}(z, n)$ in $D$ are the zeros of

$$
\operatorname{det}\left(I+F_{n}^{ \pm}(z)\right) \text {. }
$$


But (1.5) converges to $\operatorname{det}\left(I+F_{0}^{ \pm}(z)\right)$ uniformly on $D$, and by [6, Theorem 3.1], this function vanishes only at $\lambda_{0}$, and the order of its zero there is $m$. By Rouche's theorem, (1.5) must have zeros inside $D$ of total multiplicity $m$, if $n$ is large. If $\varepsilon>0$, the same argument applies to $D_{\varepsilon}=\left\{t:\left|z-\lambda_{0}\right|<\varepsilon\right\}$, so that for $n$ large, all the zeros of (1.5) in $D$ are also in $D_{\varepsilon}$; that is, the zeros of (1.5) in $D$ all converge to $\lambda_{0}$.

2. The main theorem. The following is our principal result.

2.1. TheOREM. Assume that Hypothesis III holds, and let $\zeta_{j}^{ \pm}(n)=\lambda_{j}^{ \pm}(n) \mp i \Gamma_{j}^{ \pm}(n)$ $(j=1, \ldots, m)$ be the zeros of $\operatorname{det}\left(I+F_{n}^{ \pm}(z)\right)$ in $D$, repeated according to multiplicity, and numbered in an arbitrary manner. Choose $\delta_{j}^{ \pm}(n)>0$ such that $\Gamma_{j}^{ \pm}(n)=o\left(\delta_{j}^{ \pm}(n)\right)$ and $\delta_{j}^{ \pm}(n)=o(1)$ as $n \rightarrow \infty, j=1, \ldots, m$. Define

$$
J_{n}=J_{1}^{+}(n) \cup \cdots \cup J_{m}^{+}(n) \cup J_{1}^{-}(n) \cup \cdots \cup J_{m}^{-}(n)
$$

where $J_{j}^{ \pm}(n)$ is the open interval $\left(\lambda_{j}^{ \pm}(n)-\delta_{j}^{ \pm}(n), \lambda_{j}^{ \pm}(n)+\delta_{j}^{ \pm}(n)\right)$. Then $E_{n}^{(1)}\left[J_{n}\right] \rightarrow P_{0}$ strongly.

Note that the $\zeta_{j}^{ \pm}(n)$ 's are the poles of $Q_{1}^{ \pm}(z, n)$ and hence that $\Gamma_{j}^{ \pm}(n) \geqq 0$.

The proof of Theorem 2.1 will be divided into two parts. We shall study the asymptotic behavior of $Q_{1}^{ \pm}(z, n)$ in Part $\mathrm{I}$, and give the proof of convergence in Part II. In Part I we shall, for simplicity, drop the \pm superscripts.

Part I. We shall first note two facts. In the first place, it must be shown that every subsequence of $E_{n}^{(1)}\left[J_{n}\right]$ has a subsequence converging to $P_{0}$. But since a subsequence would satisfy the same conditions as the original sequence, it suffices to prove that some subsequence of $E_{n}^{(1)}\left[J_{n}\right]$ converges to $P_{0}$.

Secondly, we may always decrease the size of $J_{n}$. For suppose that $J_{n}^{(0)} \subset J_{n}$ and $E_{n}^{(1)}\left[J_{n}^{(0)}\right] \rightarrow P_{0}$. Let $\varepsilon>0$. Then for large $n, J_{n} \subset\left(\lambda_{0}-\varepsilon, \lambda_{0}+\varepsilon\right)$, and

$$
E_{n}^{(1)}\left[J_{n}^{(0)}\right] \leqq E_{n}^{(1)}\left[J_{n}\right] \leqq E_{n}^{(1)}\left(\lambda_{0}+\varepsilon\right)-E_{n}^{(1)}\left(\lambda_{0}-\varepsilon\right) .
$$

But $E_{n}^{(1)}\left(\lambda_{0} \pm \varepsilon\right) \rightarrow E_{0}^{(1)}\left(\lambda_{0} \pm \varepsilon\right)$ by Lemma 1.3 and [8, Theorem 1.15, p. 432]. Passing to the limit in (2.1), we obtain for each $x$

$$
\begin{aligned}
\left\|P_{0} x\right\|^{2} & \leqq \lim \inf \left\|E_{n}^{(1)}\left[J_{n}\right] x\right\|^{2} \leqq \lim \sup \left\|E_{n}^{(1)}\left[J_{n}\right] x\right\|^{2} \\
& \leqq\left(E_{0}^{(1)}\left(\lambda_{0}+\varepsilon\right) x-E_{0}^{(1)}\left(\lambda_{0}-\varepsilon\right) x, x\right) .
\end{aligned}
$$

But the final term tends to $\left\|P_{0} x\right\|^{2}$ as $\varepsilon \rightarrow 0+$. Hence $\left\|E_{n}^{(1)}\left[J_{n}\right] x\right\| \rightarrow\left\|P_{0} x\right\|$, which implies that $E_{n}^{(1)}\left[J_{n}\right] \vec{s} P_{0}$, since these operators are orthogonal projections.

These facts can now be used to reduce considerably the complexity of the problem. By passing to a subsequence, we may assume that the number $N$ of distinct poles is independent of $n$. Let the poles be numbered so that $\lambda_{1}(n) \leqq \lambda_{2}(n)$ $\leqq \cdots \leqq \lambda_{N}(n)$, and $\Gamma_{k}(n)<\Gamma_{k+1}(n)$ if $\lambda_{k}(n)=\lambda_{k+1}(n)$. By again passing to a subsequence, we may then assume that the order of each pole $\zeta_{k}(n)$ is independent of $n$.

Now the open set $J_{n}$ has a finite number of connected components, and if $\lambda_{k}(n)$ and $\lambda_{r}(n)$ lie in the same component of $J_{n}$, then so do all of the $\lambda_{j}(n)$ 's between 
$\lambda_{k}(n)$ and $\lambda_{r}(n)$. Thus, for each $n$, the $\lambda_{f}(n)$ 's are partitioned into sets

$$
\left\{\lambda_{1}(n), \lambda_{2}(n), \ldots, \lambda_{p}(n)\right\}, \quad\left\{\lambda_{p+1}(n), \lambda_{p+2}(n), \ldots\right\}, \quad \ldots, \quad\left\{\ldots, \lambda_{N}(n)\right\}
$$

so that the elements of each set are those $\lambda_{f}(n)$ 's which lie in the same connected component of $J_{n}$. Since there are only a finite number of such partitions, we may assume by passing to a subsequence that the partition is independent of $n$. We shall call the sets of the corresponding partition of the poles pole clusters. Thus two poles $\zeta_{k}(n)$ and $\zeta_{r}(n)$ belong to the same pole cluster iff $\lambda_{k}(n)$ and $\lambda_{r}(n)$ belong to the same connected component of $J_{n}$.

Now consider the effect of decreasing $J_{n}$ to $J_{n}^{*}$ by replacing each $\delta_{j}(n)$ by $\delta_{j}^{*}(n)$ $\leqq \delta_{j}(n)$ such that we still have $\Gamma_{j}(n)=o\left(\delta_{j}^{*}(n)\right)(j=1, \ldots, N)$. If we again pass to a subsequence to obtain pole clusters for $J_{n}^{*}$, it is clear that the new pole clusters are subdivisions of the old; for example $\left\{\zeta_{1}(n), \zeta_{2}(n), \ldots, \zeta_{p}(n)\right\}$ may break into several $J_{n}^{*}$ pole clusters

$$
\left\{\zeta_{1}(n), \zeta_{2}(n), \ldots, \zeta_{s}(n)\right\}, \quad\left\{\zeta_{s+1}(n), \ldots\right\}, \ldots,\left\{\ldots, \zeta_{p}(n)\right\} .
$$

Since the number of poles is finite this process is eventually stable, and we obtain a $J_{n}$ for which the pole clusters are minimal in the sense that no replacement of $\delta_{j}(n)$ 's by $\delta_{j}^{*}(n)$ 's as above will lead to a nontrivial subdivision of these pole clusters.

We shall therefore assume that the pole clusters of $J_{n}$ are minimal. If $\left\{\zeta_{1}(n), \ldots, \zeta_{p}(n)\right\}$ is taken as a typical pole cluster, then the corresponding connected component is

$$
J_{1}(n) \cup \cdots \cup J_{p}(n)=\left(c_{n}-\rho_{n}, c_{n}+\rho_{n}\right) ;
$$

$c_{n}$ will be called the center and $\rho_{n}$ the radius of the pole cluster. Note that

$$
\lambda_{1}(n) \leqq c_{n} \leqq \lambda_{p}(n) .
$$

We must have

$$
\lambda_{k}(n)-c_{n}=o\left(\rho_{n}\right), \quad k=1, \ldots, p .
$$

For if we replace $\delta_{k}(n)$ by $\delta_{k}^{*}(n)$ such that in addition to the previous conditions, $\delta_{k}^{*}(n)=o\left(\delta_{k}(n)\right)(k=1, \ldots, p)$, then by minimality $\lambda_{1}(n), \ldots, \lambda_{p}(n)$ are all in the same connected component $\left(c_{n}^{*}-\rho_{n}^{*}, c_{n}^{*}+\rho_{n}^{*}\right)$ of $J_{n}^{*}$. By $(2.2), c_{n}$ is also in this component and we have

$$
\lambda_{k}(n)-c_{n}=O\left(\rho_{n}^{*}\right)=o\left(\rho_{n}\right), \quad k=1, \ldots, p .
$$

Clearly (2.3) holds for any $\lambda_{k}(n)$ if $c_{n}$ is the center of its pole cluster.

Finally, if $c_{n}(1), c_{n}(2), \rho_{n}(1)$ and $\rho_{n}(2)$ are the centers and radii of two distinct pole clusters, then we may assume that

$$
\rho_{n}(i)=o\left(\left|c_{n}(1)-c_{n}(2)\right|\right), \quad i=1,2 .
$$

For since $\left(c_{n}(1)-\rho_{n}(1), c_{n}(1)+\rho_{n}(1)\right)$ and $\left(c_{n}(2)-\rho_{n}(2), c_{n}(2)+\rho_{n}(2)\right)$ do not intersect, we have

$$
\rho_{n}(i) \leqq\left|c_{n}(1)-c_{n}(2)\right|, \quad i=1,2,
$$


while the above construction yields $c_{n}^{*}(i)$ and $\rho_{n}^{*}(i)(i=1,2)$ such that $\rho_{n}^{*}(i)$ $=o\left(\rho_{n}(i)\right), i=1,2$, and, by $(2.2)$,

$$
c_{n}^{*}(i)-c_{n}=O\left(\rho_{n}^{*}(i)\right), \quad i=1,2 .
$$

From the last three equations follows

$$
\rho_{n}^{*}(i)=o\left(\left|c_{n}^{*}(1)-c_{n}^{*}(2)\right|\right), \quad i=1,2 .
$$

The interpretation of (2.3) is that the poles are asymptotically very close to the center of the interval $\left(c_{n}-\rho_{n}, c_{n}+\rho_{n}\right)$, while (2.4) says that the components of $J_{n}$ are asymptotically very small compared with their relative distance apart.

Having made these reductions, we are ready to study the asymptotic form of the singularities of $Q_{1}^{+}(z, n)$. Let

$$
\Delta_{n}(z)=\left(z-\zeta_{1}(n)\right)^{p_{1}}\left(z-\zeta_{2}(n)\right)^{p_{2}} \cdots\left(z-\zeta_{N}(n)\right)^{p_{N}}
$$

where $p_{i}$ is the order of $\zeta_{i}(n)$. Then $\Delta_{n}(z) \rightarrow\left(z-\lambda_{0}\right)^{N}$ uniformly on $\partial D$. Write $Q_{1}^{+}(z, n)$ in a Laurent expansion

$$
Q_{1}^{+}(z, n)=P_{n}(z) / \Delta_{n}(z)+L_{n}(z)
$$

where $P_{n}(z)$ is a polynomial in $z$ with coefficients of finite rank and $L_{n}(z)$ is analytic on $D$. We assert that

$$
\lim P_{n}(z)=\left(z-\lambda_{0}\right)^{N-1} A_{0} P_{0}\left[B_{0} P_{0}\right]^{*}
$$

and

$$
\lim L_{n}(z)=L_{0}(z)
$$

uniformly on $D$. For let $P_{n}(z)=C_{p-1}^{(n)} z^{p-1}+\cdots+C_{0}(n)$. Then $C_{p-1}(n)$ is the sum of the residues of $P_{n}(z) / \Delta_{n}(z)$ and hence

$$
C_{p-1}(n)=\frac{1}{2 \pi i} \int_{\partial D} \frac{P_{n}(z)}{\Delta_{n}(z)} d z=\frac{1}{2 \pi i} \int_{\partial D} Q_{1}^{+}(z, n) d z
$$

since $L_{n}(z)$ is analytic. But $Q_{1}^{+}(z, n)$ converges to $Q_{1}^{+}(z, 0)$ uniformly on $\partial D$, so that $C_{p-1}(n)$ is a convergent sequence. Again $C_{p-2}(n)$ is the sum of the residues of $\left(\left(z-\zeta_{1}(n)\right) / \Delta_{n}(z)\right)\left(P_{n}(z)-C_{p-1}(n) z^{p-1}\right)$ so that

$$
C_{p-2}(n)=\frac{1}{2 \pi i} \int_{\partial D}\left\{Q_{1}^{+}(z, n)-\frac{\left(z-\zeta_{1}(n)\right) C_{p-1}(n) z^{p-1}}{\Delta_{n}(z)}\right\} d z
$$

where the integrand again converges uniformly on $\partial D$. Proceeding in this manner, we find that $P_{n}(z)$ is uniformly convergent on $D$. If $D_{1}$ is a slightly larger disc, concentric with $D$, then

$$
L_{n}(z)=\frac{1}{2 \pi i} \int_{\partial D_{1}} \frac{1}{\zeta-z}\left\{Q_{1}^{+}(\zeta, n)-\frac{P_{n}(\zeta)}{\Delta_{n}(\zeta)}\right\} d \zeta
$$

so that $L_{n}(z)$ also converges uniformly on $D$. (2.6) and (2.7) now follow easily from (1.4). 
We now claim that we may assume that

$$
\left\|P_{n}(z)\right\| \leqq C\left|\Delta_{n}(z)\right|(\operatorname{Im} z)^{-1}, \quad \operatorname{Im} z>0, z \in D,
$$

where $C$ is a constant independent of $n$. For by possibly decreasing $D$ and dropping a finite number of terms of the sequence, we may assume that $U_{n}(z)$ and $U_{n}^{-1}(z)$ differ from $I$ in norm by less than $(4 d)^{-1}$ on $D$, where $d=\operatorname{dim} \operatorname{ker}\left(I+Q^{+}\left(\lambda_{0}, 0\right)\right)$. Let $\Pi$ be the projection on $\operatorname{ker}\left(I+Q^{+}\left(\lambda_{0}, 0\right)\right)$, and choose $x_{1}, \ldots, x_{d} \in \mathscr{D}$ such that $\Pi x_{1}, \ldots, \Pi x_{d}$ is an orthonormal basis of $\Pi \mathscr{H}^{\prime}$. Now it is easily seen that

$$
E_{n}(z)=U_{n}(z) P_{n}(z) U_{n}^{-1}(z)
$$

is an operator on $\Pi \mathscr{H}^{\prime}$. The sets

$$
\left\{\Pi U_{n}^{-1}(z) x_{1}, \ldots, \Pi U_{n}^{-1}(z) x_{d}\right\} \text { and }\left\{\Pi U_{n}^{*}(z) x_{1}, \ldots, \Pi U_{n}^{*}(z) x_{d}\right\}
$$

are still bases of $\Pi \mathscr{H}^{\prime}$, and the matrix of $E_{n}(z)$ between these bases is

$$
\begin{aligned}
&\left(E_{n}(z) \Pi U_{n}^{-1}(z) x_{i},\right.\left.\Pi U_{n}^{*}(z) x_{j}\right) \\
&=\left(P_{n}(z) x_{i}, x_{j}\right)=\Delta_{n}(z)\left(Q_{1}^{+}(z, n) x_{i}, x_{j}\right)+\Delta_{n}(z)\left(L_{n}(z) x_{i}, x_{j}\right), \\
& i, j=1, \ldots, d,
\end{aligned}
$$

since $\Pi E_{n}(z) \Pi=E_{n}(z)$. The second term is uniformly bounded, while the first term is equal to $\Delta_{n}(z)\left(R_{n}(z) B_{n}^{*} x_{i}, A_{n}^{*} x_{j}\right)$ which does not exceed

$$
\left|\Delta_{n}(z)\right|\left|B_{n}^{*} x_{i}\right|\left|A_{n}^{*} x_{j}\right|(\operatorname{Im} z)^{-1}, \quad \operatorname{Im} z>0 .
$$

But $B_{n}^{*} x_{i}$ and $A_{n}^{*} x_{j}$ are convergent, so we may bound the matrix in (2.9) by $C_{1}\left|\Delta_{n}(z)\right|(\operatorname{Im} z)^{-1}$. A little consideration of the bases used shows that this implies that

$$
\left\|E_{n}(z)\right\| \leqq C_{2}\left|\Delta_{n}(z)\right|(\operatorname{Im} z)^{-1}, \quad \operatorname{Im} z>0,
$$

and (2.8) follows easily.

We are now ready to study the partial fraction expansion of $P_{n}(z) / \Delta_{n}(z)$. Consider the first pole cluster $\zeta_{1}(n), \ldots, \zeta_{p}(n)$, with center $c_{1}(n)$ and radius $\rho_{1}(n)$. By the binomial series

$$
\left(z-\zeta_{1}(n)\right)^{-1}=\left[z-c_{1}(n)-\left(\zeta_{1}(n)-c_{1}(n)\right)\right]^{-1}=\left(z-c_{1}(n)\right)^{-1}\{1+o(1)\}
$$

uniformly on $\left|z-c_{1}(n)\right| \geqq \rho_{1}(n)$, by (2.4). The portion of the partial fraction expansion of $P_{n}(z) / \Delta_{n}(z)$ involving these poles can therefore be written as

$$
\frac{P_{n}^{(1)}(z)}{\left(z-\zeta_{1}(n)\right)^{m_{1}} \cdots\left(z-\zeta_{p}(n)\right)^{m_{p}}}=\frac{P_{n}^{(1)}(z)\{1+o(1)\}}{\left(z-c_{1}(n)\right)^{p_{1}}}
$$

uniformly on $\left|z-c_{1}(n)\right| \geqq \rho_{1}(n)$, where $p_{1}=m_{1}+\cdots+m_{p}$. Expanding the right side of (2.10) in partial fractions, we obtain

$$
\left\{\frac{A_{p_{1}}^{(1)}(n)}{\left(z-c_{1}(n)\right)^{p_{1}}}+\cdots+\frac{A_{1}^{(1)}(n)}{\left(z-c_{1}(n)\right)}\right\}\{1+o(1)\}
$$


uniformly on $\left|z-c_{1}(n)\right| \geqq \rho_{1}(n)$. There are analogous expansions for the terms corresponding to the other pole clusters. We wish to estimate the coefficients $A_{j}^{(k)}(n)$ in these expansions, and the key to this is (2.8). Define

$$
\alpha_{j}^{(k)}(n)=(-i)^{j}\left(\rho_{k}(n)\right)^{-j+1} A_{j}^{(k)}(n)
$$

for $1 \leqq j \leqq p_{k}, k=1, \ldots, N$, where $N$ is the number of pole clusters. Set $z=c_{1}(n)$ $+i \rho_{1}(n) \sigma^{-1}$ in (2.8), where $\frac{1}{2} \leqq \sigma \leqq 1$. Then the above expansions are valid, and the term (2.11) becomes

$$
\left(\rho_{1}(n)\right)^{-1}\left\{\alpha_{1}^{(1)}(n) \sigma+\cdots+\alpha_{p_{1}}^{(1)}(n) \sigma^{p_{1}}\right\}\{1+o(1)\}
$$

uniformly on $\frac{1}{2} \leqq \sigma \leqq 1$. For the same value of $z$, the terms of $(2.8)$ for the other pole clusters are of the form

$$
\frac{A_{j}^{(k)}(n)}{\left(z-c_{k}(n)\right)^{j}}=\frac{(i)^{j} \alpha_{j}^{(k)}(n)}{\rho_{1}(n)} \frac{\rho_{1}(n)\left(\rho_{k}(n)\right)^{j-1}}{\left(z-c_{k}(n)\right)^{j}} .
$$

However, by (2.4),

$$
\frac{\rho_{1}(n)\left(\rho_{k}(n)\right)^{j-1}}{\left(z-c_{k}(n)\right)^{j}} \sim \frac{\rho_{1}(n)\left(\rho_{k}(n)\right)^{j-1}}{\left(c_{1}(n)-c_{k}(n)\right)^{j}}=o(1) .
$$

(2.8) therefore yields the equation

$$
\left\{\alpha_{1}^{(1)}(n)+\cdots+\alpha_{p_{1}}^{(1)}(n) \sigma^{p_{1}-1}\right\}+\sum_{k=2}^{N} \sum_{j=1}^{p_{1}}\left\{\alpha_{j}^{(k)}(n) o(1)\right\}=O(1)
$$

uniformly on $\frac{1}{2} \leqq \sigma \leqq 1$. There is an analogous equation for each of $N$ pole clusters. From these equations we form a system of $p_{1}+\cdots+p_{N}$ linear equations for the $p_{1}+\cdots+p_{N}$ quantities $\alpha_{j}^{(k)}(n)$ in the following manner: evaluate the analogue of (2.12) for the $k$ th pole cluster at $p_{k}$ distinct points of $\frac{1}{2} \leqq \sigma \leqq 1$, yielding $p_{k}$ equations. If this is done for each $k$, we obtain $p_{1}+\cdots+p_{N}$ equations. If we let $\alpha_{n}$ be the "unknown" vector, and $\beta_{n}$ the inhomogeneous term, then these equations in matrix form become

$$
\left(D+M_{n}\right) \alpha_{n}=\beta_{n}
$$

where $M_{n}$ consists of the $o(1)$ terms, and hence vanishes as $n \rightarrow \infty$, and $\beta_{n}=O(1)$. $D$ is independent of $n$, and consists of $N$ blocks $D_{1}, \ldots, D_{N}$ on the diagonal, where

$$
D_{k}\left(\alpha_{1}^{(k)}, \ldots, \alpha_{p_{k}}^{(k)}\right)=\left(\varphi_{k}\left(\sigma_{1}\right), \ldots, \varphi_{k}\left(\sigma_{p_{k}}\right)\right)
$$

$\varphi_{k}(\sigma)=\alpha_{1}^{(k)}+\cdots+\alpha_{p_{k}}^{(k)} \sigma^{p_{k}-1}$, and $\sigma_{1}, \ldots, \sigma_{p_{k}}$ are distinct points. But if a polynomial of degree $p_{k}-1$ vanishes at $p_{k}$ distinct points it is zero. Therefore $D_{1}, \ldots, D_{N}$ and hence $D$ are invertible. For large $n$ we thus obtain

$$
\alpha_{n}=\left(D+M_{n}\right)^{-1} \beta_{n}=O(1)
$$

by the Neumann series. This means that the $\alpha_{j}^{(k)}(n)$ 's are all bounded, and hence that

$$
A_{j}^{(k)}(n)=O\left(\left[\rho_{k}(n)\right]^{-j+1}\right)
$$


for $j=1, \ldots, p_{k}$, and $k=1, \ldots, N$. Moreover, since $\rho_{k}(n)$ could be replaced by $\rho_{k}^{*}(n)=o\left(\rho_{k}(n)\right)$, we have for $j \geqq 2$,

$$
A_{j}^{(k)}(n)=O\left(\left[\rho_{k}^{*}(n)\right]^{-j+1}\right)=o\left(\left[\rho_{k}(n)\right]^{-j+1}\right) .
$$

Let $\gamma_{k}^{+}(n)$ be the upper half of $\left|z-c_{k}(n)\right|=\rho_{k}(n), \gamma_{k}^{-}(n)$ the lower half, both positively oriented, and $\gamma_{k}(n)=\gamma_{k}^{+}(n)+\gamma_{k}^{-}(n)$. By elementary estimates, using (2.4) and (2.15), we find that for any analytic function $\Phi(z)$ on $D$

$$
\int_{\gamma_{1}^{+}(n)} \Phi(z)\left(z-c_{k}(n)\right)^{-j} A_{j}^{(k)}(n)\{1+o(1)\} d z=o(1)
$$

unless $k=j=1$, in which case, using (2.14),

$$
\begin{aligned}
\int_{\gamma_{1}^{+}(n)} \Phi(z)\left(z-c_{1}(n)\right)^{-1} A_{1}^{(1)}(n)\{1+o(1)\} d z \\
=i \pi \Phi\left(c_{1}(n)\right) A_{1}^{(1)}(n)+o(1) \\
=\frac{1}{2} \int_{\gamma_{1}(n)} \Phi(z)\left(z-c_{1}(n)\right)^{-1} A_{1}^{(1)}(n)\{1+o(1)\} d z
\end{aligned}
$$

since $\Phi(z)-\Phi\left(c_{1}(n)\right)=o(1)$ uniformly on $\gamma_{1}^{+}(n)$.

Part II. Let us first observe that the reductions to minimal pole clusters could have been made for the poles of $Q_{1}^{+}(z, n)$ and $Q_{1}^{-}(z, n)$ simultaneously. We shall assume that this has been done. Of course, all the poles in a given cluster may or may not lie on the same side of the axis. Thus

Define

$$
J_{n}=\bigcup_{k=1}^{N}\left(c_{k}(n)-\rho_{k}(n), c_{k}(n)+\rho_{k}(n)\right) \text {. }
$$

$$
J_{n}^{ \pm}= \pm\left(\gamma_{1}^{ \pm}(n)+\cdots+\gamma_{N}^{ \pm}(n)\right)
$$

where $\gamma_{k}^{-}(n)$ is the positively oriented lower half of $\left|z-c_{k}(n)\right|=\rho_{k}(n)$. Let $\varphi, \psi \in C_{c}^{\infty}(R)$ be functions which agree with polynomials on $D \cap R$, and let $x, y \in \mathscr{D}$. It is easy to see that

$$
\begin{aligned}
& \left(E_{n}^{(1)}\left[J_{n}\right] \varphi\left(H_{n}\right) B_{n}^{*} x, \psi\left(H_{n}\right) A_{n}^{*} y\right) \\
& \quad=\frac{1}{2 \pi i} \int_{J_{n}^{+}} \varphi(z) \psi(z)\left(Q_{1}^{+}(z, n) x, y\right) d z-\frac{1}{2 \pi i} \int_{J_{n}^{-}} \varphi(z) \psi(z)\left(Q_{1}^{-}(z, n) x, y\right) d z
\end{aligned}
$$

where $\varphi(z)$ and $\bar{\psi}(z)$ are the polynomials equal to $\varphi(\lambda)$ and $\psi(\lambda)$ for real $\lambda \in D$. The terms in (2.18) corresponding to $L_{n}^{ \pm}(z)$ are $o(1)$ since $L_{n}^{ \pm}(z)$ is uniformly bounded. Hence, by (2.16) and (2.17) we find that the first integral of (2.18) is equal to

$$
\frac{1}{2} \sum_{k=1}^{N} \varphi\left(c_{k}(n)\right) \bar{\psi}\left(c_{k}(n)\right)\left(A_{1}^{(k)}(n) x, y\right)+o(1)=\frac{1}{4 \pi i} \int_{\partial D} \varphi(z) \psi(z) \frac{\left(P_{n}^{+}(z) x, y\right)}{\Delta_{n}^{+}(z)} d z+o(1) .
$$

By (2.6), this converges to

$$
\frac{1}{4 \pi i} \int_{\partial D} \varphi(z) \bar{\psi}(z) \frac{\left(P_{0} B_{0}^{*} x, A_{0}^{*} y\right)}{z-\lambda_{0}} d z=\frac{\varphi\left(\lambda_{0}\right) \bar{\psi}\left(\lambda_{0}\right)}{2}\left(P_{0} B_{0}^{*} x, A_{0}^{*} y\right) .
$$


By a similar analysis, the second term of (2.18) converges to the same quantity, so that we finally obtain

$$
\lim _{n \rightarrow \infty}\left(E_{n}^{(1)}\left[J_{n}\right] \varphi\left(H_{n}\right) B_{n}^{*} x, \psi\left(H_{n}\right) A_{n}^{*} y\right)=\left(P_{0} \varphi\left(H_{0}\right) B_{0}^{*} x, \psi\left(H_{0}\right) A_{0}^{*} y\right)
$$

for $x, y \in \mathscr{D}$, and $\varphi, \psi$ as described.

Let $P_{n}=E_{n}^{(1)}\left[J_{n}\right], x_{n}=\varphi\left(H_{n}\right) B_{n}^{*} x$ and $y_{n}=\psi\left(H_{n}\right) A_{n}^{*} y$. By Lemma 1.3 above and Lemma 5.1 of [4], $\varphi\left(H_{n}\right) \rightarrow \varphi\left(H_{0}\right)$ strongly, so that $x_{n} \rightarrow x_{0}$ strongly, and similarly $y_{n} \rightarrow y_{0}$ strongly. Hence,

$$
\left(P_{n} x_{0}, y_{0}\right)-\left(P_{0} x_{0}, y_{0}\right)=\left(P_{n}\left(x_{0}-x_{n}\right), y_{0}\right)+\left(P_{n} x_{n}, y_{0}-y_{n}\right)+\left(P_{n} x_{n}, y_{n}\right)-\left(P_{0} x_{0}, y_{0}\right)
$$

tends to zero, by (2.19). Moreover [6, $\S 1]$ elements of the form $x_{0}$, and those of the form $y_{0}$ are dense in the reducing subspace $\mathscr{M}_{0}$, of $T_{0}$ and $H_{0}$ referred to in Theorem 1.2 , so that

$$
\left(P_{n} x, y\right) \rightarrow\left(P_{0} x, y\right), \quad x, y \in \mathscr{M}_{0} .
$$

Let $y \in \mathscr{M}_{0}^{\perp}$. Then for every $\varepsilon>0$

$$
\left|P_{n} y\right| \leqq\left|E_{n}^{(1)}\left(\lambda_{0}+\varepsilon\right) y-E_{n}^{(1)}\left(\lambda_{0}-\varepsilon\right) y\right| .
$$

By $[8$, Theorem 1.15, p. 432]

$$
\lim \sup \left|P_{n} y\right| \leqq\left|E_{0}^{(1)}\left(\lambda_{0}+\varepsilon\right) y-E_{0}^{(1)}\left(\lambda_{0}-\varepsilon\right) y\right|=\left|E_{0}\left(\lambda_{0}+\varepsilon\right) y-E_{0}\left(\lambda_{0}-\varepsilon\right) y\right| .
$$

But this last expression vanishes as $\varepsilon \rightarrow 0+$, since $\lambda_{0}$ is not an eigenvalue of $T_{0}$. Thus

$$
P_{n} y \rightarrow P_{0} y
$$

strongly, for $y \in \mathscr{M}_{0}^{\perp}$. Finally, let $u=x+y, x \in \mathscr{M}_{0}, y \in \mathscr{M}_{0}^{\perp}$, be an arbitrary element of $\mathscr{H}$. Then

$$
\left|P_{n} u\right|^{2}=\left(P_{n} x, x\right)+\left(P_{n} y, x+y\right)+\left(x, P_{n} y\right) .
$$

The last two terms vanish by $(2.21)$, so that by $(2.20)$

$$
\left|P_{n} u\right|^{2} \rightarrow\left|P_{0} x\right|^{2}=\left|P_{0} u\right|^{2}
$$

which implies that $P_{n} \rightarrow P_{0}$.

3. Unperturbed operators of finite rank. We are now in a position to improve the results in $\$ 5$ of [5]. Let $H_{0}$ be a selfadjoint operator of finite rank $r, T_{1}$ a selfadjoint operator, and define $H_{\varepsilon}=H_{0}+\varepsilon T_{1}$ for $\varepsilon \geqq 0$. If we take $T_{\varepsilon}=\varepsilon T_{1}$, and make the factorization $H_{0}=H_{0} P$, where $P$ is the orthogonal projection on the range of $H_{0}$, then $G_{\varepsilon}(z)=\varepsilon^{-1} G_{1}(z / \varepsilon)$ so that $Q_{\varepsilon}(z)=\varepsilon^{-1} \Phi(z / \varepsilon)$ where $\Phi(z)=H_{0} G_{1}(z) P$ is an operator on the finite-dimensional range of $H_{0}$. Thus, $I+Q_{\varepsilon}(z)$ is the WeinsteinAronszajn matrix

$$
W(z, \varepsilon)=I+H_{0} G_{\varepsilon}(z) P=I+\varepsilon^{-1} \Phi(z / \varepsilon)
$$


for the perturbation of $\varepsilon T_{1}$ by $H_{0}$. We assume as usual that the smallest subspace reducing $T_{1}$ and containing the range of $H_{0}$ is the whole space $\mathscr{H}$.

If $\delta>0$ and $0<\alpha<\pi / 2$, define $\Sigma(\alpha, \delta)$ to be the union of the two "sectors" $\{z:|z|>\delta$ and $|\arg z|<\alpha\}$ and $\{z:|z|>\delta$ and $|\arg z-\pi|<\alpha\}$. Our Basic Hypothesis $[5, \S 1]$ is that $\Phi(z)$ has a continuation $\Phi_{+}(z)$ from $\operatorname{Im} z>0$ to some $\Sigma\left(\alpha_{0}, \delta_{0}\right)$. In order that our theory apply, we must show that as $\varepsilon \rightarrow 0+$,

$$
\varepsilon^{-1} \Phi_{+}(z / \varepsilon) \rightarrow-z^{-1} H_{0}=H_{0} G_{0}(z) P
$$

uniformly on some neighborhood $\Omega$ of the nonzero eigenvalues $c_{1}, \ldots, c_{r}$ of $H_{0}$. This is shown to hold in Theorem 2 of [5], under the additional assumption that

$$
\Phi_{+}(z)-\Phi_{-}(z)=O\left(z^{-2}\right)
$$

uniformly on $\Sigma\left(\alpha_{0}, \delta_{0}\right)$, where $\Phi_{-}(z)$ is the continuation of $\Phi(z)$ to $\Sigma\left(\alpha_{0}, \delta_{0}\right)$ from $\operatorname{Im} z<0 . \Omega$ is taken to be $\Sigma\left(\alpha_{0}, \delta_{1}\right)$ where $0<\delta_{1}<\min \left\{\left|c_{1}\right|, \ldots,\left|c_{r}\right|\right\}$. The other conditions of Hypothesis III are easily verified. This yields

3.1. THEOREM. Under the hypotheses of Theorem 2 of [5], there is spectral concentration on the union of the intervals $\left(\lambda_{k}(\varepsilon)-\delta_{k}(\varepsilon), \lambda_{k}(\varepsilon)+\delta_{k}(\varepsilon)\right)$, where $z_{k}(\varepsilon)=\lambda_{k}(\varepsilon)$ $-i \Gamma_{k}(\varepsilon)(k=1, \ldots, r), \Gamma_{k}(\varepsilon)=o\left(\delta_{k}(\varepsilon)\right)$, and $\delta_{k}(\varepsilon)=o(1)$, as $\varepsilon \rightarrow 0+$.

This generalizes Theorem 5 of [5], in which we assumed that the perturbation $\varepsilon T_{1}$ removed the degeneracy of $H_{0}$, and also that the left side of (3.1) was $O\left(z^{-3}\right)$.

4. Barrier penetration problems for ordinary differential operators. We now wish to apply the theory to some problems of quantum mechanical "barrier penetration." According to Beck and Nussenzweig, "complex eigenvalues" were first introduced into quantum mechanics in connection with such problems, in the famous paper [2] of Gamov on alpha-particle decay. The usual textbook approach is via the WKB method.

As usually stated, the problem contains no natural small parameter, or unperturbed operator, so we will have to formulate it in a way in which concentration becomes meaningful. We shall therefore consider an operator

$$
H_{0}=-d^{2} / d x^{2}+q(x)+\beta^{2}
$$

where $q(x) \in L_{1} . H_{0}$ will have continuous spectrum $\left[\beta^{2}, \infty\right)$ and perhaps some eigenvalues in $\left(-\infty, \beta^{2}\right)$. We shall assume that $H_{0}$ has an eigenvalue $\lambda_{0}, 0<\lambda_{0}<\beta^{2}$. If we now "cut off" the potential $q(x)+\beta^{2}$ at a large distance $R$ from the origin to obtain the operator

$$
H_{R}=-d^{2} / d x^{2}+\left[q(x)+\beta^{2}\right] \chi_{[0, R]}(x)
$$

then we find that $H_{R}$ has continuous spectrum $[0, \infty)\left(\chi_{[0, R]}\right.$ is the characteristic function of $[0, R])$. The eigenvalue $\lambda_{0}$ has disappeared. However, if the barrier width $R$ becomes large, we can expect concentration of the spectrum of $H_{R}$ at $\lambda_{0}$. If we choose $T_{0}$ and $T_{R}$ to be the operators obtained by setting $q(x)=0$ above, then 
$T_{0}$ and $T_{R}$ are computable and our method applies to show that there is concentration due to a virtual pole.

We shall consider both the one-dimensional Schroedinger operator on $(-\infty,+\infty)$ and the radial equation on $(0, \infty)$.

EXAMPLE 1. The radial equation, $l=0$. Let $q(x) \in L_{1}(0, \infty)$ be real valued and not identically zero, and $\beta>0$. Define

$$
H_{0}=-d^{2} / d x^{2}+q(x)+\beta^{2}
$$

and

$$
T_{0}=-d^{2} / d x^{2}+\beta^{2} .
$$

For $R>0$, let $\chi_{[0, R]}(x)$ denote the characteristic function of $[0, R]$ and define

$$
H_{R}=-d^{2} / d x^{2}+\left[\beta^{2}+q(x)\right] \chi_{[0, R]}(x)
$$

and

$$
T_{R}=-d^{2} / d x^{2}+\beta^{2} \chi_{[0, R]}(x) .
$$

All of these operators are given the boundary condition $u(0)=0$, and operate on $L_{2}(0, \infty)$. We shall choose $A_{0}, B_{0}, A_{R}$ and $B_{R}$ to be multiplication operators:

$$
\begin{array}{ll}
A_{0}=|q(x)|^{1 / 2}, & B_{0}=\operatorname{sgn} q(x)|q(x)|^{1 / 2}, \\
A_{R}=|q(x)|^{1 / 2} \chi_{[0, R]}(x), & B_{R}=\operatorname{sgn} q(x)|q(x)|^{1 / 2} \chi_{[0, R]}(x) .
\end{array}
$$

Note that if $q(x)$ has compact support, then for $R$ sufficiently large $A_{R}=A_{0}$ and $B_{R}=B_{0}$.

4.1. THEOREM. If $\lambda_{0}$ is an eigenvalue of $H_{0}, 0<\lambda_{0}<\beta^{2}$, and $q(x) \in L_{1}(0, \infty)$, then as $R \rightarrow \infty$, the above operators satisfy the conditions of Theorem 2.1.

We shall proceed to verify the conditions of Hypothesis III. First of all, $\mathscr{D}\left(T_{R}\right)$ $=\mathscr{D}\left(T_{0}\right)=\mathscr{D}\left(d^{2} / d x^{2}\right)$, since $T_{R}$ and $T_{0}$ differ from $-d^{2} / d x^{2}$ by bounded operators. But $\mathscr{D}\left(d^{2} / d x^{2}\right)$ contains only bounded functions (cf. [8, p. 301]). Thus $\mathscr{D}\left(A_{R}\right)$ $=\mathscr{D}\left(B_{R}\right) \supseteq \mathscr{D}\left(T_{R}\right)$ for $R \geqq 0$, and Hypothesis I(a) clearly holds, since $A_{R}$ and $B_{R}$ are selfadjoint and commute.

Secondly, $\sigma\left(T_{0}\right)=\left[\beta^{2}, \infty\right)$, so that $\lambda_{0}$ cannot be an eigenvalue of $T_{0}$. Moreover, $\mathscr{D}\left(A_{0}\right) \subseteq \mathscr{D}\left(A_{R}\right)$ and $A_{R} f \rightarrow A_{0} f$ strongly for $f \in \mathscr{D}\left(A_{0}\right)$ (Hypotheses III(c) and (e)). Since $T_{R}-T_{0}$ is bounded and converges strongly to zero, as $R \rightarrow \infty$, we have for $\operatorname{Im} z \neq 0$

$$
\begin{aligned}
\left\|G_{R}(z) f-G_{0}(z) f\right\| & =\left\|G_{R}(z)\left(T_{0}-T_{R}\right) G_{0}(z) f\right\| \\
& \leqq|\operatorname{Im} z|^{-1}\left\|\left(T_{0}-T_{R}\right) G_{0}(z) f\right\|
\end{aligned}
$$

for each $f \in L_{2}(0, \infty)$. If we show that for some nonreal $z_{0}$

$$
\left\|A_{R} G_{0}\left(z_{0}\right)\right\| \leqq C
$$


where $C$ is independent of $R$, then for each $f \in L_{2}(0, \infty)$

$$
A_{R} G_{R}\left(z_{0}\right) f-A_{0} G_{0}\left(z_{0}\right) f=A_{R} G_{0}\left(z_{0}\right)\left(T_{0}-T_{R}\right) G_{R}\left(z_{0}\right) f+\left(A_{R}-A_{0}\right) G_{0}\left(z_{0}\right) f
$$

vanishes strongly as $R \rightarrow \infty$, for $G_{0}\left(z_{0}\right) f \in \mathscr{D}\left(A_{R}\right) \subseteq \mathscr{D}\left(A_{0}\right)$, and $A_{R}-A_{0}$ and $T_{R}-T_{0}$ vanish strongly. Hypothesis III(d) then follows, since ' $B_{R}=W A_{R}$, where $W=\operatorname{sgn} q(x)$ is bounded. To prove $(4.5)$, note that $[1, \mathrm{p} .1329]\left(T_{0}-z\right)^{-1}$ is an integral operator with Green's function kernel

$$
\begin{aligned}
G_{0}(x, y ; k) & =-\kappa^{-1} \sinh \kappa x e^{-\kappa y}, & & y>x, \\
& =-\kappa^{-1} \sinh \kappa y e^{-\kappa x}, & & y<x .
\end{aligned}
$$

Therefore, if $k=i \eta, \eta>0$, then $\kappa=\left(\beta^{2}+\eta^{2}\right)^{1 / 2}>0$ and

$$
\left|G_{0}(x, y ; k)\right| \leqq(2 \kappa)^{-1} \exp (-\kappa|x-y|) .
$$

It follows that the Schmidt norm of the kernel

$$
|q(x)|^{1 / 2} \chi_{[0, R]}(x) G_{0}(x, y ; k)
$$

does not exceed

$$
(2 \kappa)^{-1} \int_{0}^{\infty} \int_{0}^{\infty}|q(x)| \exp (-\kappa|x-y|) d x d y \leqq \kappa^{-2} \int_{0}^{\infty}|q(x)| d x
$$

which is finite, since $q \in L_{1}(0, \infty)$. This proves (4.5). This estimate also shows that $A_{0} G_{0}\left(-\eta^{2}\right)$ is compact, and hence by formula (1.1), that $R_{0}(z)-G_{0}(z)$ is compact. Therefore, $\sigma_{e}\left(H_{0}\right)=\sigma_{e}\left(T_{0}\right)=\left[\beta^{2}, \infty\right)$, where $\sigma_{e}$ denotes essential spectrum, so that $\lambda_{0}$ is an isolated eigenvalue of finite (in fact, simple) multiplicity. This fact is well known, and can be deduced in a number of ways (cf. [3, §4] and [1, pp. 1593-1604]).

In general, if $\operatorname{Re} \kappa>0,(4.6)$ may be replaced by

$$
\left|G_{0}(x, y ; k)\right| \leqq(2|\kappa|)^{-1} \exp (-\operatorname{Re} \kappa|x-y|)
$$

and it follows that $A_{0} G_{0}(z) B_{0}$ is analytic in Schmidt norm in the $z$-plane, cut along the interval $\left[\beta^{2}, \infty\right)=\sigma\left(T_{0}\right)$.

Let $\beta^{2}>\lambda_{0} \in \sigma\left(T_{0}\right)$, and let $P$ be the projection on $\operatorname{ker}\left(H_{0}-\lambda_{0}\right)$. The range of $P$ is contained in $\mathscr{D}\left(H_{0}\right)=\mathscr{D}\left(T_{0}\right) \subseteq \mathscr{D}\left(A_{0}\right)=\mathscr{D}\left(B_{0}\right)$, and the residue of $A_{0} R_{0}(z) B_{0}$ is $A P[B P]^{*}\left[6\right.$, Theorem 3.1]. If $A P[B P]^{*}=0$, then multiplying by $\operatorname{sgn} q(x)$, we obtain $B P[B P]^{*}=0$, and hence $B P=0$. Thus if $\varphi \in \operatorname{ker}\left(H_{0}-\lambda_{0}\right)$, we have $B \varphi=0$, or, after multiplying by $|q(x)|^{1 / 2}, q(x) \varphi(x)=0$ a.e. But $\varphi(x)$ is continuously differentiable, so if $q(x) \neq 0$ on a set of positive measure, then $\varphi(x)$ vanishes on a set of positive measure. But such a set has an accumulation point $x_{0}$, and hence $\varphi\left(x_{0}\right)$ $=\varphi^{\prime}\left(x_{0}\right)=0$. It follows that $\varphi(x)$ vanishes identically, since $\varphi$ satisfies

$$
-\varphi^{\prime \prime}(x)+q(x) \varphi(x)=\left(\lambda_{0}-\beta^{2}\right) \varphi(x) .
$$

Therefore, if $\lambda_{0}$ is a nontrivial eigenvalue of $H_{0}, A_{0} R_{0}(z) B_{0}$ has a nontrivial pole at $\lambda_{0}$, unless $q(x)$ vanishes identically. 
It remains to consider $Q_{R}(z)=A_{R} G_{R}(z) B_{R}$. For this, we shall require the Green's function kernel for $G_{R}(z)=\left(T_{R}-z\right)^{-1}$. This is given by [1, p. 1329].

$$
\begin{aligned}
G_{R}(x, y ; k) & =[W(k)]^{-1} \psi_{+}(x, k) \varphi(y, k), & & y<x, \\
& =[W(k)]^{-1} \psi_{+}(y, k) \varphi(x, k), & & y>x,
\end{aligned}
$$

where $z=k^{2}, \operatorname{Im} k>0 . \psi_{+}(x, k)$ and $\varphi(x, k)$ are the solutions of

$$
-u^{\prime \prime}(x)+\beta^{2} \chi_{[0, R]}(x) u(x)=k^{2} u(x)
$$

such that $\varphi(0, k)=0$ and $\psi_{+}(x, k)$ is square-integrable at infinity, while

$$
W(k)=\left|\begin{array}{cc}
\varphi(x, k) & \psi_{+}(x, k) \\
\varphi^{\prime}(x, k) & \psi_{+}^{\prime}(x, k)
\end{array}\right|
$$

is the Wronskian of $\psi_{+}$and $\varphi$, which is independent of $x$. Elementary calculations yield

$$
\begin{aligned}
\psi_{+}(x, k) & =a e^{-x x}+b e^{x x}, & & 0 \leqq x \leqq R, \\
& =e^{i k x}, & & R \leqq x,
\end{aligned}
$$

where $\kappa^{2}=\beta^{2}-k^{2}, \operatorname{Im} \kappa>0$ and

$$
a=(2 \kappa)^{-1}(\kappa-i k) e^{\kappa R} e^{i k R}
$$

and

$$
b=(2 \kappa)^{-1}(\kappa+i k) e^{-\kappa R} e^{i k R}
$$

while

$$
\begin{aligned}
\varphi(x, k) & =\sinh \kappa x, & & 0 \leqq x \leqq R, \\
& =c e^{i k x}+d e^{-i k x}, & & R \leqq x,
\end{aligned}
$$

where

$$
c=(4 i k)^{-1} e^{-i k R}\left[(\kappa+i k) e^{\kappa R}+(\kappa-i k) e^{-\kappa R}\right]
$$

and

$$
d=-(4 i k)^{-1} e^{i k R}\left[(\kappa-i k) e^{\kappa R}+(\kappa+i k) e^{-\kappa R}\right] .
$$

Computing the Wronskian for $x=0$, we obtain $W(k)=-\kappa(a+b)$.

Observe now that (4.7) and the formulas following it provide a continuation of $G_{R}(x, y ; k)$ across the interval $(0, \beta)$ to the lower half of the $k$-plane. Let $\Omega$ be a bounded neighborhood of $\lambda_{0}, 0<\lambda_{0}<\beta$, which is bounded away from $k=\beta$ and $k=0$. Since $\kappa$ is real and positive on $(-\infty, \beta)$ (and pure imaginary on $(\beta, \infty)$ ), we may choose $\Omega$ such that $\operatorname{Re} \kappa$ is positive and bounded away from zero on $\Omega$. Let $k \in \Omega$ and $0 \leqq x \leqq R ;$ then

$$
[W(k)]^{-1} \psi_{+}(x, k)=O\left(e^{-x x}\right) .
$$


This may be seen by multiplying both $W(k)$ and $\psi_{+}(x, k)$ by $e^{-\kappa R} e^{-t k R}$. Then $e^{-\kappa R} e^{-t k R} W(k)$ is bounded, and bounded away from zero, and $e^{-\kappa R} e^{-i k R} \psi_{+}(x, k)$ $=O\left(e^{-\kappa x}\right)$. Since $\sinh \kappa x=O\left(e^{\kappa x}\right)$, we have that for $0 \leqq y<x \leqq R$ and $k \in \Omega$,

$$
G_{R}(x, y ; k)=O\left(e^{-\kappa x} e^{\kappa y}\right)=O(1),
$$

and similarly for $0 \leqq x<y \leqq R$. Hence

$$
\sup _{0 \leqq x, y \leqq R}\left|G_{R}(x, y ; k)\right| \leqq C, \quad k \in \Omega,
$$

where $C$ is independent of $R$ and $k \in \Omega$.

Now, for $k \in \Omega$, define $Q^{+}\left(k^{2}\right)$ to be the operator with kernel

$$
\chi_{[0, R]}(x)|q(x)|^{1 / 2} G_{R}(x, y ; k)|q(y)|^{1 / 2} \operatorname{sgn} q(y) \chi_{[0, R]}(y) .
$$

This kernel is analytic in $k$, and we have

$$
\begin{aligned}
\left\|Q^{+}\left(k^{2}\right)\right\|_{2}^{2} & =\int_{0}^{R} \int_{0}^{R}|q(x)|\left|G_{R}(x, y)\right|^{2}|q(y)| d x d y \\
& \leqq C^{2}\left(\int_{0}^{\infty}|q(x)| d x\right)^{2}
\end{aligned}
$$

for the Schmidt norm of $Q^{+}\left(k^{2}\right)$. It follows that $Q^{+}\left(k^{2}\right)$ is a compact, analytic extension of $Q\left(k^{2}\right)$ to a neighborhood of $\lambda_{0}$. Thus Hypothesis II holds. Hypothesis $\mathrm{I}(\mathrm{b})$ is verified similarly. For Hypothesis $\operatorname{III}(\mathrm{a})$, note that $\chi_{[0, R]}(x) G_{R}(x, y ; k) \chi_{[0, R]}(y)$ converges pointwise to $G_{0}(x, y ; k)$ and is uniformly bounded. By the above estimates $Q_{R}^{+}\left(k^{2}\right) \rightarrow Q_{0}^{+}\left(k^{2}\right)$ in Schmidt norm. This completes the verification of the conditions.

EXAMPLE 2. The one-dimensional Schroedinger equation. Let $q(x) \in L_{1}(-\infty,+\infty)$ be real valued and not identically zero, and $\beta>0$. Define $H_{0}$ and $T_{0}$ by (4.1) and (4.2), and let

$$
H_{R}=-d^{2} / d x^{2}+\left[\beta^{2}+q(x)\right] x_{[-R,+R]}(x)
$$

and

$$
T_{R}=-d^{2} / d x^{2}+\beta^{2} \chi_{[-R,+R]}(x)
$$

for $R>0$. In this case, there are no boundary conditions. $A_{0}, B_{0}, A_{R}$ and $B_{R}$ are given by (4.3)-(4.4).

4.2. THEOREM. If $\lambda_{0}$ is an eigenvalue of $H_{0}, 0<\lambda_{0}<\beta^{2}$, and $q(x) \in L_{1}(-\infty,+\infty)$, then as $R \rightarrow \infty$, the above operators satisfy the conditions of Theorem 2.1 .

The proof is essentially the same as that of Theorem 4.1. We shall simply note the appropriate formulas for $G_{0}$ and $G_{R}$.

Let $\zeta=k^{2}, \operatorname{Im} k>0$ and $\kappa^{2}=\beta^{2}-k^{2}, \operatorname{Re} \kappa>0$. Then

$$
G_{0}(x, y ; k)=-\kappa^{-1} \exp (-\kappa|x-y|)
$$

while

$$
\begin{array}{rlr}
G_{R}(x, y ; k) & =[W(k)]^{-1} \psi_{+}(x, k) \psi_{-}(y, k), & y<x, \\
& =[W(k)]^{-1} \psi_{+}(y, k) \psi_{-}(x, k), & y>x,
\end{array}
$$


where $W(k)$ is the Wronskian of $\psi_{+}$and $\psi_{-}$and $\psi_{ \pm}(x, k)$ satisfies

$$
-\psi_{ \pm}^{\prime \prime}(x)+\beta^{2} \chi_{[-R,+R]}(x) \psi_{ \pm}(x)=k^{2} \psi_{ \pm}(x)
$$

and is square-integrable at $\pm \infty$. By symmetry, $\psi_{-}(x)=\psi_{+}(-x)$, and $\psi_{+}$is the same as in the preceding example:

$$
\begin{aligned}
\psi_{+}(x, k) & =a e^{-\kappa x}+b e^{\kappa x}, & & -R \leqq x \leqq+R, \\
& =e^{i k x}, & & R \leqq x,
\end{aligned}
$$

where $a$ and $b$ are given by (4.9) and (4.10). We do not need $\psi_{+}(x, k)$ for $x \leqq-R$. We may now compute

$$
G_{R}(x, y ; k)=\left[2 \kappa\left(b^{2}-a^{2}\right)\right]^{-1}\left[a e^{-\kappa x}+b e^{+\kappa x}\right]\left[a e^{+\kappa y}+b e^{-\kappa y}\right]
$$

for $-R \leqq y<x \leqq R$. It now follows easily that $G_{R}(x, y ; k)$ is bounded on $[-R, R]$ $\times[-R, R]$ uniformly in $R$, and that $G_{R}(x, y ; k) \rightarrow G_{0}(x, y ; k)$ as $R \rightarrow+\infty$.

Notes Added in Proof. (1) In Theorem 2.1, the poles of $Q_{1}^{-}(z, n)$ are the complex conjugates of the poles of $Q_{1}^{+}(z, n)$, at least when either $A$ or $B$ is bounded, and perhaps in general. Thus, in this case, $J_{k}^{+}(n)$ and $J_{k}^{-}(n)$ are the same if $\delta_{k}^{+}(n)=\delta_{k}^{-}(n)$.

(2) The obscure formula just following (2.13) signifies that the action of $D_{k}$ on the vector $\left(\alpha_{1}^{(k)}, \ldots, \alpha_{p_{k}}^{(k)}\right)$ produces a vector whose components are the values of the polynomial $\varphi_{k}$ at the points $\sigma_{1}, \ldots, \sigma_{p_{k}}$.

\section{REFERENCES}

1. N. Dunford and J. T. Schwartz, Linear operators. I: General theory, Pure and Appl. Math., vol. 7, Interscience, New York, 1958. MR 22 \#8302.

- Linear operators. II: Spectral theory. Selfadjoint operators in Hilbert space, Interscience, New York, 1963. MR 32 \#6181.

2. G. Gamov, Zur quanten Theorie des Atomkernes, Z. Phys. 51 (1928), 204.

3. J. S. Howland, Banach space techniques in the perturbation theory of self-adjoint operators with continuous spectra, J. Math. Anal. Appl. 20 (1967), 22-47. MR 36 \#2011.

4. - Perturbation of embedded eigenvalues by operators of finite rank, J. Math. Anal. Appl. 23 (1968), 575-584. MR 37 \#5723.

5. - Spectral concentration and virtual poles, Amer. J. Math. 91 (1969), 1106-1126. MR 40 \#7863.

6. - On the Weinstein-Aronszajn formula, Arch. Rational Mech. Anal.39(1970), 323-339.

7. T. Kato, Wave operators and similarity for some non-selfadjoint operators, Math. Ann. 162 (1965/66), 258-279. MR 32 \#8211.

8. — , Perturbation theory for linear operators, Die Grundlehren der math. Wissenschaften, Band 132, Springer-Verlag, New York, 1966. MR 34 \#3324.

9. R. Konno and S. T. Kuroda, On the finiteness of perturbed eigenvalues, J. Fac. Sci. Univ. Tokyo Sect. I 13 (1966), 55-63. MR 34 \#1848.

10. M. Ribarič and I. Vidav, Analytic properties of the inverse $A(z)^{-1}$ of an analytic linear operator valued function A(z), Arch. Rational Mech. Anal. 32 (1969), 298-310. MR 38 \#5035.

11. S. Steinberg, Meromorphic families of compact operators, Arch. Rational Mech. Anal. 31 (1968/69), 372-379. MR 38 \#1562.

University of Virginia, Charlottesville, Virginia 22903 